\title{
Low Temperature Expansions for the Gibbs States of Weakly Interacting Quantum Ising Lattice Systems
}

\author{
Lawrence E. Thomas ${ }^{1}$ and Zhong Yin \\ Department of Mathematics, University of Virginia, Charlottesville, VA 22903, USA
}

\begin{abstract}
Low temperature expansions for the Gibbs states of weakly interacting transverse Ising-like models are developed, by conditioning the states on a sub-algebra of observables. The conditioned states have effective classical Hamiltonians which are estimated by the solution to a simple implicit equation. Provided the interaction is sufficiently weak but fixed independent of temperature, and the temperature is sufficiently low, exponential clustering of the correlation functions holds.
\end{abstract}

\section{Introduction}

Let $H_{\Lambda}(\varepsilon)$ be the transverse Ising Hamiltonian associated with a finite volume $\Lambda \subset \mathbb{Z}^{\nu}$

$$
H_{\Lambda}(\varepsilon)=-\sum_{i \in \Lambda} \sigma^{x}(i)-\varepsilon \sum_{A \subset A} \hat{V}_{\Lambda}(A) \sigma^{z}(A)
$$

Here, $\sigma^{z}(A)=\bigotimes_{i \in A} \sigma^{z}(i)$ and $\sigma^{x}(i)$ and $\sigma^{z}(i)$ are the Pauli spin matrices acting at the site $i$

$$
\sigma^{x}(i)=\left(\begin{array}{ll}
0 & 1 \\
1 & 0
\end{array}\right), \quad \sigma^{z}(i)=\left(\begin{array}{rr}
1 & 0 \\
0 & -1
\end{array}\right),
$$

taken in a basis so the first term of the Hamiltonian is a spin-flip term, the second a classical Ising term. To simplify the analysis, we assume that $H_{\Lambda}(\varepsilon)$ is translation invariant, i.e. that $\Lambda$ is rectangular and that periodic boundary conditions are imposed. Finally, we assume that the coefficients $\hat{V}_{A}(A)$, which are real, are equal to zero for the cardinality of $A,|A|$, exceeding some constant $C$, independent of $\Lambda$.

The purpose of this article is to show if $\varepsilon$ is fixed and sufficiently small, then the Gibbs state corresponding to $H_{\Lambda}(\varepsilon)$,

$$
\langle X\rangle_{\Lambda, \varepsilon, \beta}=\left(\operatorname{tr} \exp \left(-\beta H_{\Lambda}(\varepsilon)\right)\right)^{-1} \operatorname{tr} X \exp \left(-\beta H_{\Lambda}(\varepsilon)\right)
$$

1 Work partially supported by NSF-MCS 74-07313-A 03 
exhibits clustering of correlation functions in the thermodynamic limit, at sufficiently low temperature; in particular, the truncated correlation functions

$$
\lim _{\Lambda \uparrow \mathbb{Z}^{v}}\left\langle\sigma^{z}(A) \sigma^{z}(B+x)\right\rangle_{\Lambda, \beta, \varepsilon}^{T}
$$

decay exponentially fast as $x \rightarrow \infty$, for $\beta \equiv T^{-1}>\beta_{0}(\varepsilon)$.

The physical intuition for this result is simply that quantum fluctuations caused by the spin-flip terms of the Hamiltonian overwhelm any tendency of the Ising terms to correlate the spins at remote sites. The result can be regarded as a partial converse to that of Ginibre [1] who, for example, shows that if the Ising term is nearest neighbor ferromagnetic, then long range order obtains at low temperature, provided $\varepsilon$ is sufficiently large. (Ginibre's estimates actually require that $\varepsilon \rightarrow \infty$ for $T \rightarrow 0$ for long range order; this restriction has been removed by Kirkwood [2] who shows long range order for $T>T_{0}$ and $\varepsilon>\varepsilon_{0}$, with $\varepsilon_{0}$ independent of $T_{0}$, by a reflection positivity argument.)

The strategy for proof is a familiar one; we render the quantum Gibbs state $\langle\cdot\rangle_{\Lambda}$ into a classical Ising model state in one higher dimension, the extra dimension corresponding to the "time" $t, 0 \leqq t \leqq \beta$. However, we do not employ the Trotter product formula. Rather, we condition at a set of times $0, \tau_{0}, 2 \tau_{0}, \ldots,(N-1) \tau_{0}$ with $\tau_{0}=\beta / N, N$ an appropriately chosen integer. Define $h_{\Lambda}\left(\sigma^{1}, \sigma^{2}, \tau, \varepsilon\right)$ by the transfer matrix expression

$$
\exp \left(-\frac{1}{2} h_{\Lambda}\left(\sigma^{1}, \sigma^{2}, \tau, \varepsilon\right)\right)=\left\langle\sigma^{1}\left|\exp -\tau H_{\Lambda}(\varepsilon)\right| \sigma^{2}\right\rangle .
$$

Here, $\sigma^{1}, \sigma^{2} \in\{-1,1\}^{4}$ are classical Ising configurations; as ortho-normal basis for the Hilbert space $\bigotimes_{A} \mathbb{C}^{2}$ with the usual inner product, we take the set of vectors $|\sigma\rangle=\bigotimes_{i \in \Lambda}|\sigma(i)\rangle$ with $|\sigma(i)\rangle$ an eigenstate at site $i$ which is spin up or down with respect to $\sigma^{z}(i)$ according to whether $\sigma(i)$ is +1 or -1 . With this notation, we have that if $X(0, \sigma), X\left(\tau_{0}, \sigma\right), \ldots, X\left((N-1) \tau_{0}, \sigma\right)$ are multiplication operators at times $0, \tau_{0}, \ldots,(N-1) \tau_{0}$, then

$$
\begin{aligned}
\left\langle X(0) \ldots X\left((N-1) \tau_{0}\right)\right\rangle= & \left(\mathbb{Z}_{\Lambda}(\varepsilon)\right)^{-1} \sum_{\sigma^{1}, \ldots, \sigma^{N}}\left(X\left(0, \sigma^{0}\right) \ldots X\left((N-1) \tau_{0}, \sigma^{N-1}\right)\right) \\
& \cdot \exp \left(-\frac{1}{2} \sum_{i=1}^{N} h_{\Lambda}\left(\sigma^{i-1}, \sigma^{i}, \tau_{0}, \varepsilon\right)\right)
\end{aligned}
$$

where $\mathbb{Z}_{\Lambda}(\varepsilon)$ is the partition function and $\sigma^{0} \equiv \sigma^{N}$, i.e. the expectation can be written as a classical Ising expression. Provided that $h_{A}$ is small in the appropriate sense one can then apply high temperature Ising expansion methods [3-7] to establish the clustering property.

How do we make $h_{\Lambda}\left(\sigma^{1}, \sigma^{2}, \tau_{0}, \varepsilon\right)$ appropriately small? Clearly if $\tau_{0}$ is very small, $\sigma^{1}$ and $\sigma^{2}$ are highly correlated so that $h_{\Lambda}$ cannot be small. On the other hand our estimate for $h_{\Lambda}\left(\sigma^{1}, \sigma^{2}, \tau_{0}, \varepsilon\right)$, which we obtain as the solution to a differential equation, breaks down for large $\tau_{0}$. The idea, then, is to choose $\tau_{0}$ to be an intermediate time so that the correlations between $\sigma^{1}$ and $\sigma^{2}$ can relax but the effects of the perturbation, terms of $O(\varepsilon)$, have not had sufficient time to grow too 
large. That such a choice for $\tau_{0}$ (and $\varepsilon$ ) can be made is the main content of this article.

We note that the estimate for $h_{A}$ (actually its local density) is in terms of a function which is defined implicitly and which has been analyzed in detail by Hagedorn and Rafelski [8]. This estimating function also arises in an expansion for the ground state of $H_{A}(\varepsilon)$ [9]; in fact, the analysis presented here is to be regarded as a time-dependent version of the ground state case.

The (classical) high temperature Ising expansions referred to above provide bounds on the decay of the correlation functions Eq. (1.4) which are uniform in $N$ [recall $N$ is the number of factors contained in Eq. (1.6)]. For this reason, we obtain exponential clustering of correlations for a sequence of temperatures defined by $\beta=N \tau_{0}, N=1,2, \ldots$. Since, for a given $\varepsilon$ suitably small, $\tau_{0}$ can range over an interval, it follows that clustering holds at all sufficiently low temperatures.

We conclude with miscellaneous remarks. First, although it appears that one can only compute expectations of observables at the times $0, \tau_{0}, 2 \tau_{0}, \ldots,(N-1) \tau_{0}$, this is actually not the case. By adding a (possibly time-dependent) perturbation $\lambda W$ to the Hamiltonian $H_{A}$ and then differentiating an expectation with respect to $\lambda$, one could in principle compute, for example, a Duhamel correlation function.

The second remark is that the method is applicable to other spin models, e.g. a discrete rotator model with $\sigma(i)=(\cos (2 \pi m / n), \sin (2 \pi m / n)), m=0,1, \ldots, n-1$ and with the spin-flip term of the Hamiltonian Eq. (1.1) replaced by a sum of discrete Laplacians on the unit circle. For the case $\sigma(i)= \pm 1$, it is of interest to replace the spin-flip terms of Eq. (1.1) by terms of the form $\sigma^{x}(i) \sigma^{x}(j)$ for example. The resulting Hamiltonian is, up to a constant factor, unitarily equivalent (dual) to a Hamiltonian of the form Eq. (1.1) with quadratic Ising terms [9]. An expansion for this modified model analogous to the expansion described here would provide a proof (alternative to that of $[1,2]$ ) of long range order at low temperature for the ferromagnetic quadratic Ising term model Eq. (1.1), at large $\varepsilon$. This work will be described elsewhere. For a general proof of long range order in quantum Ising systems relying on reflection positivity (see [10]).

Finally, we remark that other more heuristic results concerning the critical behavior and critical indices for transverse Ising models can be found in the work of Elliott et al., for example, [11]. The one-dimensional nearest neighbor transverse Ising model can be solved explicitly at zero temperature (see [12]).

\section{Weak Interaction Expansion}

We introduce the following notation:

1) Let $\left.f \in C\{-1,1\}^{\Lambda} \times\{-1,1\}^{\Lambda}\right)$ and

$$
f\left(\sigma^{\prime}, \sigma\right)=\sum_{A, B \subset A} \hat{f}(A, B) \sigma^{\prime}(A) \sigma(B),
$$

where $\sigma^{\prime}, \sigma \in\{-1,1\}^{A}$ and $C(X)$ is the space of continuous functions on $X$ and $\sigma(A)=\prod_{i \in A} \sigma(i)$. Then we define the cardinality of $f$ to be $C(f)=\sup _{\substack{A, B \subset A \\ f(A, B) \neq 0}}|B|$, the range of $f$ to be

$$
r(f)=\sup _{\substack{A, B \subset A \\ f(A, B) \neq 0}} \sup _{i, j \in B}|i-j|
$$


and the super range of $f$ to be

$$
R(f)=\sup _{\substack{A, B \subset A \\ f(A, B) \neq 0}} \sup _{i, j \in A \cup B}|i-j|
$$

where $|i-j|=\sum_{k=1}^{v}\left|i_{k}-j_{k}\right|$. In particular, if $f \in C\left(\{-1,1\}^{\Lambda}\right)$ and $f(\sigma)=\sum_{A \subseteq A} \hat{f}(A) \sigma(A)$, then we define the cardinality of $f$ to be $C(f)=\sup _{\substack{A \subset A \\ \hat{f}(A) \neq 0}}|A|$ and the range of $f$ to be $r(f)=\sup _{\substack{A \subset A \\ f(A) \neq 0}} \sup _{i, j \in A}|i-j|$, and the super range of $f, R(f)$, to be $r(f)$.

2) Let $f$ be given by Eq. (2.1). Define

$$
f^{m}\left(\sigma^{\prime}, \sigma\right)=\sum_{\substack{A, B \subset A \\|B|=m}} \hat{f}(A, B) \sigma^{\prime}(A) \sigma(B) .
$$

We call each term of $f^{m}\left(\sigma^{\prime}, \sigma\right)$ an $m$-term of $f\left(\sigma^{\prime}, \sigma\right)$. Define the $n o r m\|\cdot\|_{0}$ by

$$
\|f\|_{0}=\sum_{A, B \subset A}|\hat{f}(A, B)| .
$$

It is obvious that

$$
\|f\|_{0} \geqq\|f\|_{\infty}=\sup _{\sigma, \sigma^{\prime}}\left|f\left(\sigma^{\prime}, \sigma\right)\right| .
$$

3) Let $h_{A}\left(\sigma^{\prime}, \sigma, t, \varepsilon\right)$ be defined in Eq. (1.5). By a Perron-Frobenius argument, $\left\langle\sigma^{\prime}\left|e^{-t H_{\Lambda}(\varepsilon)}\right| \sigma\right\rangle>0$ for all $t>0$, so $h_{\Lambda}$ is well-defined. Define

$$
h_{\Lambda}\left(i, \sigma^{\prime}, \sigma, t, \varepsilon\right)=\frac{1}{2}\left(h_{\Lambda}\left(\sigma^{\prime}, \sigma, t, \varepsilon\right)-h_{\Lambda}\left(\sigma^{\prime}, \sigma_{i}, t, \varepsilon\right)\right) \text {, }
$$

where $\sigma_{i}(j)=\sigma(j)$ if $j \neq i$ and $\sigma_{i}(i)=-\sigma(i)$.

4) Define an operator $K$ on $C\left(\{-1,1\}^{\Lambda} \times\{-1,1\}^{\Lambda}\right)$ by

$$
K f\left(\sigma^{\prime}, \sigma\right)=\sum_{B \neq \emptyset}|B| \tilde{f}\left(\sigma^{\prime}, B\right) \sigma(B)+\tilde{f}\left(\sigma^{\prime}, \emptyset\right),
$$

for $f\left(\sigma^{\prime}, \sigma\right)=\sum_{B \subset A} \tilde{f}\left(\sigma^{\prime}, B\right) \sigma(B),(\emptyset$ is the empty set). Then we have

$$
\begin{aligned}
f\left(\sigma^{\prime}, \sigma\right) & =\sum_{B \subset \Lambda} \tilde{f}\left(\sigma^{\prime}, B\right) \sigma(B)=\sum_{i \in \Lambda}\left(\sum_{B \ni i} \frac{1}{|B|} \tilde{f}\left(\sigma^{\prime}, B\right) \sigma(B)+\frac{\tilde{f}\left(\sigma^{\prime}, \emptyset\right)}{|\Lambda|}\right) \\
& =K^{-1} \sum_{i \in \Lambda}\left(f\left(i, \sigma^{\prime}, \sigma\right)+f^{0}\left(i, \sigma^{\prime}\right)\right),
\end{aligned}
$$

where $f\left(i, \sigma^{\prime}, \sigma\right)=\sum_{B \ni i} \tilde{f}\left(\sigma^{\prime}, B\right) \sigma(B)=\frac{1}{2}\left(f\left(\sigma^{\prime}, \sigma\right)-f\left(\sigma^{\prime}, \sigma_{i}\right)\right)$ and $f^{0}\left(i, \sigma^{\prime}\right)=\tilde{f}\left(\sigma^{\prime}, \emptyset\right) /|\Lambda|$.

Remark. Note that $f\left(i, \sigma^{\prime}, \sigma\right)$ has the following important property (closure property) [9]. If $f\left(i, \sigma^{\prime}, \sigma\right)$ and $f\left(j, \sigma^{\prime}, \sigma\right)$ have terms $\tilde{f}^{\prime}\left(\sigma^{\prime}, B\right) \sigma(B)$ and $\tilde{f}^{\prime \prime}\left(\sigma^{\prime}, B\right) \sigma(B)$, respectively, then $\tilde{f}^{\prime}\left(\sigma^{\prime}, B\right)=\tilde{f}^{\prime \prime}\left(\sigma^{\prime}, B\right)$. 
It is easy to see that

where

$$
\begin{aligned}
\frac{\partial}{\partial t}\left\langle\sigma^{\prime}\left|e^{-t H_{\Lambda}(\varepsilon)}\right| \sigma\right\rangle & =\left\langle\sigma^{\prime}\left|e^{-t H_{\Lambda}(\varepsilon)}\left(-H_{\Lambda}(\varepsilon)\right)\right| \sigma\right\rangle \\
& =\sum_{i \in \Lambda}\left\langle\sigma^{\prime}\left|e^{-t H_{\Lambda}(\varepsilon)} \sigma^{x}(i)\right| \sigma\right\rangle+\varepsilon\left\langle\sigma^{\prime}\left|e^{-t H_{\Lambda}(\varepsilon)}\right| \sigma\right\rangle V_{\Lambda}(\sigma),
\end{aligned}
$$

$$
V_{\Lambda}(\sigma)=\left\langle\sigma\left|\sum_{A \subset \Lambda} \hat{V}_{\Lambda}(A) \sigma^{z}(A)\right| \sigma\right\rangle=\sum_{A \subset \Lambda} \hat{V}_{\Lambda}(A) \sigma(A) .
$$

For the moment, we omit the subscript $\Lambda$. By Eq. (1.5), Eq. (2.7) can be written as

$$
-\frac{1}{2} \frac{\partial}{\partial t} h\left(\sigma^{\prime}, \sigma, t, \varepsilon\right)=\sum_{i \in \Lambda} \exp h\left(i, \sigma^{\prime}, \sigma, t, \varepsilon\right)+\varepsilon V(\sigma) .
$$

Using identity (2.6), we rewrite Eq. (2.8a) as

$$
-\frac{1}{2} K^{-1} \frac{\partial}{\partial t} \sum_{i \in \Lambda}\left(h\left(i, \sigma^{\prime}, \sigma, t, \varepsilon\right)+h^{0}\left(i, \sigma^{\prime}, t, \varepsilon\right)\right)=\sum_{i \in \Lambda} \exp h\left(i, \sigma^{\prime}, \sigma, t, \varepsilon\right)+\varepsilon V(\sigma) .
$$

Letting $K$ act on both sides of Eq. (2.8b), we obtain

$$
-\frac{1}{2} \frac{\partial}{\partial t} \sum_{i \in \Lambda}\left(h\left(i, \sigma^{\prime}, \sigma, t, \varepsilon\right)+h^{0}\left(i, \sigma^{\prime}, t, \varepsilon\right)\right)=K\left(\sum_{i} \exp h\left(i, \sigma^{\prime}, \sigma, t, \varepsilon\right)+\varepsilon V(\sigma)\right) .
$$

Since $\Lambda$ is a finite set, $h\left(i, \sigma^{\prime}, \sigma, t, \varepsilon\right)$ and $h^{0}\left(i, \sigma^{\prime}, t, \varepsilon\right)$ are analytic in $\varepsilon$ for small $\varepsilon$. Then we have

$$
h\left(i, \sigma^{\prime}, \sigma, t, \varepsilon\right)=\sum_{n=0}^{\infty} \varepsilon^{n} h_{n}\left(i, \sigma^{\prime}, \sigma, t\right)
$$

and

$$
h^{0}\left(i, \sigma^{\prime}, t, \varepsilon\right)=\sum_{n=0}^{\infty} \varepsilon^{n} h_{n}^{0}\left(i, \sigma^{\prime}, t\right)
$$

An easy calculation shows that

$$
\begin{aligned}
\exp h_{0}\left(i, \sigma^{\prime}, \sigma, t\right) & =\left\langle\sigma^{\prime}\left|e^{t \sum_{j} \sigma^{x}(j)} \sigma^{x}(i)\right|\right\rangle\left\langle\left\langle\sigma^{\prime}\left|e^{t \sum_{J} \sigma^{x}(j)}\right| \sigma\right\rangle\right. \\
& =\left(\cosh 2 t-\sigma^{\prime}(i) \sigma(i)\right) / \sinh 2 t .
\end{aligned}
$$

Substituting these expansions for $h$ and $h^{0}$ into Eq. (2.8c) and equating coefficients of $\varepsilon^{n}$, we get

$$
-\frac{1}{2} \frac{\partial}{\partial t} \sum_{i}\left(h_{1}\left(i, \sigma^{\prime}, \sigma, t\right)+h_{1}^{0}\left(i, \sigma^{\prime}, t\right)\right)=K\left[\sum_{i}\left(\exp h_{0}\left(i, \sigma^{\prime}, \sigma, t\right)\right) h_{1}\left(i, \sigma^{\prime}, \sigma, t\right)+V(\sigma)\right],
$$

and

$$
\begin{aligned}
-\frac{1}{2} \sum_{i} \frac{\partial}{\partial t}\left(h_{n}\left(i, \sigma^{\prime}, \sigma, t\right)+h_{n}^{0}\left(i, \sigma^{\prime}, t\right)\right)= & K\left[\sum _ { i } ( \operatorname { e x p } h _ { 0 } ( i , \sigma ^ { \prime } , \sigma , t ) ) \left(h_{n}\left(i, \sigma^{\prime}, \sigma, t\right)\right.\right. \\
& \left.\left.+P_{n}\left(h_{1}\left(i, \sigma^{\prime}, \sigma, t\right), \ldots, h_{n-1}\left(i, \sigma^{\prime}, \sigma, t\right)\right)\right)\right],
\end{aligned}
$$


for $n \geqq 2$, where $P_{n}\left(X_{1}, \ldots, X_{n-1}\right)$ is the polynomial defined by

$$
\exp \left(\varepsilon X_{1}+\varepsilon^{2} X_{2}+\ldots\right)=1+\sum_{n=1}^{\infty} \varepsilon^{n}\left(X_{n}+P_{n}\left(X_{1}, \ldots, X_{n-1}\right)\right) .
$$

Theorem 2.1. Assume that $r\left(V_{A}\right), C\left(V_{A}\right)$, and $\left\|V_{A}\right\|_{0}|\Lambda|^{-1}$ are finite and independent of 1. Then for every $\delta>0$, there exist $\tau>0$ and $\varepsilon_{0}>0$ such that for all $t \in[\tau, 2 \tau]$ and $|\varepsilon| \in\left[0, \varepsilon_{0}\right]$;

i) the expansion for $h$ in powers of $\varepsilon$ is absolutely convergent; moreover

$$
\sum_{n=0}^{\infty} \varepsilon^{n}\left\|h_{\Lambda n}\left(i, \sigma^{\prime}, \sigma, t\right)\right\|_{0}<\delta
$$

for all $i \in \Lambda$ and the inequality holds uniformly in $\Lambda$;

ii)

$$
\left\|h_{\Lambda^{\prime}}\left(i, \sigma^{\prime}, \sigma, t, \varepsilon\right)-h_{\Lambda}\left(i, \sigma^{\prime}, \sigma, t, \varepsilon\right)\right\|_{0}=O\left(\varepsilon^{d(i, \partial \Lambda) / R}\right)
$$

for all $i \in \Lambda \subset \Lambda^{\prime}$; where $d(i, \partial \Lambda)$ is the distance between $i$ and $\partial \Lambda$ and $R=r\left(V_{\Lambda}\right)$.

iii)

$$
\sum_{j \in \mathbb{Z}^{v}} \sum_{n \geqq \frac{|i-j|}{R}} \varepsilon^{n}\left\|\mathrm{~h}_{\Lambda n}\left(\mathrm{i}, \sigma^{\prime}, \sigma, \mathrm{t}\right)\right\|_{0} \exp (|i-j|)<\infty
$$

for all $i \in \Lambda$, and the series converges uniformly in $\Lambda$.

In fact, the results of our theorem imply exponential decay of the two point function [5]. Therefore, we have

Corollary. For $\beta \geqq 3 \tau$ and $|\varepsilon|<\varepsilon_{0}$, and some $\alpha>0$,

$$
\sum_{i \in \mathbb{Z}^{\nu}}\left|\left\langle\sigma^{z}(0) \sigma^{z}(i)\right\rangle_{\beta, \varepsilon}-\left\langle\sigma^{z}(0)\right\rangle_{\beta, \varepsilon}\left\langle\sigma^{z}(i)\right\rangle_{\beta, \varepsilon}\right| \exp \alpha|i|<\infty
$$

and the series converges uniformly in $\beta$. Here $\langle\cdot\rangle_{\beta, \varepsilon}=\lim _{\Lambda^{\uparrow} \mathbb{Z}^{v}}\langle\cdot\rangle_{\Lambda, \beta, \varepsilon}$ defined by Eq. (1.3).

Proof of the Theorem. In outline the proof of the theorem proceeds through several lemmas. Lemma 2.2 below specifies the initial conditions satisfied by the $h_{n}$ 's, $t \rightarrow 0$. The differential equations satisfied by the $h_{n}$ 's are all of the same form [see Eq. (2.22) below]. Lemmas 2.3 and 2.5 provide estimates on the cardinality, range and norm of the solution of Eq. (2.22) given the cardinality, range and norm of the "source" term. Lemmas 2.4 and 2.6 apply these results to the $h_{n}$ 's to obtain estimates for their cardinality, range and norm. Lemma 2.7 then shows that the series for $h\left(i, \sigma^{\prime}, \sigma, t, \varepsilon\right)$ is bounded term by term by an implicity defined analytic function.

Lemma 2.2. For all integers $m \geqq 0$ and $n \geqq 1$

Moreover, for $m>0$

$$
\lim _{t \rightarrow 0} h_{n}^{m}\left(i, \sigma^{\prime}, \sigma, t\right)=0
$$

$$
\lim _{t \rightarrow 0}\left(\sinh ^{m} 2 t\right) h^{m}\left(i, \sigma^{\prime}, \sigma, t, \varepsilon\right)=0
$$


Proof. We have that

$$
\begin{aligned}
\left\langle\sigma^{\prime}\left|e^{-t H(\varepsilon)}\right| \sigma\right\rangle= & \exp \left(-\frac{1}{2} h\left(\sigma^{\prime}, \sigma, t, \varepsilon\right)\right) \\
= & \exp \left[-\frac{1}{2} K^{-1} \sum_{i \in \Lambda}\left(h\left(i, \sigma^{\prime}, \sigma, t, \varepsilon\right)+h^{0}\left(i, \sigma^{\prime}, t, \varepsilon\right)\right)\right] \\
= & \exp \left[-\frac{1}{2} K^{-1} \sum_{i \in \Lambda}\left(\sum_{n=1}^{\infty}\left(\varepsilon^{n} h_{n}\left(i, \sigma^{\prime}, \sigma, t\right)+\varepsilon^{n} h_{n}^{0}\left(i, \sigma^{\prime}, t\right)\right)\right.\right. \\
& \left.\left.+h_{0}\left(i, \sigma^{\prime}, \sigma, t\right)+h_{0}^{0}\left(i, \sigma^{\prime}, t\right)\right)\right] .
\end{aligned}
$$

This implies that

$$
\begin{gathered}
\exp \left[-\frac{1}{2} K^{-1} \sum_{i \in \Lambda} \sum_{n=1}^{\infty} \varepsilon^{n} h_{n}\left(i, \sigma^{\prime}, \sigma, t\right)+\varepsilon^{n} h_{n}^{0}\left(i, \sigma^{\prime}, t\right)\right] \\
=\left\langle\sigma^{\prime}\left|e^{-t H(\varepsilon)}\right| \sigma\right\rangle /\left\langle\sigma^{\prime}\left|e^{-t H(0)}\right| \sigma\right\rangle \rightarrow 1, \quad t \rightarrow 0,
\end{gathered}
$$

as can be seen by expanding both numerator and denominator in powers of $t$; both numerator and denominator are of the form $(+t)^{p} / p !+O\left(t^{p+1}\right)$, where $p$ is the minimal number of flips required to convert $\sigma$ to $\sigma^{\prime}$. Hence, we have, for all $n \geqq 1, \sigma^{\prime}$, and $\sigma$,

$$
\sum_{i \in \Lambda} h_{n}\left(i, \sigma^{\prime}, \sigma, t\right)+h_{n}^{0}\left(i, \sigma^{\prime}, t\right) \rightarrow 0, \quad t \rightarrow 0 .
$$

By the closure property, we obtain, for all $m \geqq 0$

Since

$$
h_{n}^{m}\left(i, \sigma^{\prime}, \sigma, t\right) \rightarrow 0, \quad t \rightarrow 0 .
$$

$$
h_{0}\left(i, \sigma^{\prime}, \sigma, t\right)=\log \frac{\cosh 2 t-\sigma^{\prime}(i) \sigma(i)}{\sinh 2 t}
$$

Equation (2.19) follows.

Lemma 2.3. Let $k \in C\left(\{-1,1\}^{\Lambda} \times\{-1,1\}^{\Lambda}\right)$ and $k\left(i, \sigma^{\prime}, \sigma, t\right), k^{0}\left(i, \sigma^{\prime}, t\right)$ be defined as in Eq. (2.6). Consider the differential equation with $S\left(\sigma^{\prime}, \sigma, t\right)$ a source term and $k\left(i, \sigma^{\prime}, \sigma, t\right), k^{0}\left(i, \sigma^{\prime}, t\right)$ satisfying the initial conditions $k\left(i, \sigma^{\prime}, \sigma, 0\right)=0$ and $k^{0}\left(i, \sigma^{\prime}, 0\right)=0$ for all $\sigma^{\prime}$ and $\sigma$,

$$
-\frac{1}{2} \frac{\partial}{\partial t}\left(\sum_{i \in \Lambda} k\left(i, \sigma^{\prime}, \sigma, t\right)+k^{0}\left(i, \sigma^{\prime}, t\right)\right)=K\left[\sum_{i \in \Lambda}\left(\exp h_{0}\left(i, \sigma^{\prime}, \sigma, t\right)\right) k\left(i, \sigma^{\prime}, \sigma, t\right)+S\left(\sigma^{\prime}, \sigma, t\right)\right] .
$$

Then, i) $k(i, \cdot, \cdot, t)$ and $S$ have the same cardinality and range, i.e. $C(k)=C(S)$ and $r(k)=r(S)$;

ii) $R(k) \leqq R(S)$.

Proof. i) It is obvious that $C(S) \leqq C(k)$ and $r(S) \leqq r(k)$. Assume that $C(S)<C(k)$ $=m \leqq|\Lambda|$. Then, from Eq. (2.22)

$$
-\frac{1}{2} \frac{\partial}{\partial t} \sum_{i} k^{m}\left(i, \sigma^{\prime}, \sigma, t\right)=m(\operatorname{coth} 2 t) \sum_{i} k^{m}\left(i, \sigma^{\prime}, \sigma, t\right) .
$$


Equation (2.23) must hold for each term in the Fourier expansion. By the closure property, we get

$$
\frac{\partial}{\partial t} k^{m}\left(i, \sigma^{\prime}, \sigma, t\right)+2 m(\operatorname{coth} 2 t) k^{m}\left(i, \sigma^{\prime}, \sigma, t\right)=0 .
$$

Multiplying $\sinh ^{m} 2 t$ on both sides of (2.24), we get

$$
\frac{\partial}{\partial t}\left(\left(\sinh ^{m} 2 t\right) k^{m}\left(i, \sigma^{\prime}, \sigma, t\right)\right)=0 \text {. }
$$

Then $\left(\sinh ^{m} 2 t\right) k^{m}\left(i, \sigma^{\prime}, \sigma, t\right)$ is constant in $t$. But since $k^{m}\left(i, \sigma^{\prime}, \sigma, 0\right)=0$ by assumption, $k^{m}\left(i, \sigma^{\prime}, \sigma, t\right) \equiv 0$, which contradicts $C(k)=m$. Thus $C(S)=C(k)$. By the same method, we obtain $r(S)=r(k)$.

ii) Suppose $C(S)=m \geqq 1$. By part i) of the lemma, $C(k)=m$. Thus if we only consider the $m$-terms in Eq. (2.22), we have

$$
-\frac{1}{2} \frac{\partial}{\partial t} \sum_{i} k^{m}\left(i, \sigma^{\prime}, \sigma, t\right)=m\left(\sum_{i} k^{m}\left(i, \sigma^{\prime}, \sigma, t\right) \operatorname{coth} 2 t+S^{m}\left(\sigma^{\prime}, \sigma, t\right)\right) .
$$

By incorporating the initial conditions, Eq. (2.25) can be written in integral form,

$$
\sum_{i} k^{m}\left(i, \sigma^{\prime}, \sigma, t\right)=-\frac{2 m}{\sinh ^{m} 2 t} \int_{0}^{t} S^{m}\left(\sigma^{\prime}, \sigma, t^{\prime}\right) \sinh ^{m} 2 t^{\prime} d t^{\prime} .
$$

Expanding both sides of this equation, we have

$$
\sum_{i} \sum_{\substack{B \ni i, A \\|B|=m}} \hat{k}^{m}(A, B, t) \sigma^{\prime}(A) \sigma(B)=-\frac{2 m}{\sinh ^{m} 2 t} \int_{0}^{t} \sinh ^{m} 2 t^{\prime} \sum_{\substack{A^{\prime}, B^{\prime} \\\left|B^{\prime}\right|=m}} \hat{S}^{m}\left(A^{\prime}, B^{\prime}, t^{\prime}\right) \sigma^{\prime}\left(A^{\prime}\right) \sigma\left(B^{\prime}\right) d t^{\prime} .
$$

Equating coefficients of $\sigma^{\prime}(A) \sigma(B)$ on both sides of Eq. (2.27) and using the closure property, we get

$$
m \hat{k}^{m}(A, B, t)=-\frac{2 m}{\sinh ^{m} 2 t} \int_{0}^{t} \hat{S}^{m}\left(A, B, t^{\prime}\right) \sinh ^{m} 2 t^{\prime} d t^{\prime}
$$

Hence the diameter of $A \cup B \leqq R(S)$ and $R\left(k^{m}(i, \cdot, \cdot, t)\right) \leqq R(S)$ for all $i \in \Lambda$.

Let $n$ be an integer such that $0 \leqq n \leqq m-2$. Suppose $R\left(k^{m-n}(i, \cdot, \cdot, t)\right) \leqq R(S)$ for all $i \in \Lambda$. Since, by considering $(m-(n+1))$-terms in Eq. (2.22),

$$
\begin{aligned}
& -\frac{1}{2} \frac{\partial}{\partial t} \sum_{i} k^{m-n-1}\left(i, \sigma^{\prime}, \sigma, t\right) \\
& =(m-n-1) \sum_{j}\left[k^{m-n-1}\left(j, \sigma^{\prime}, \sigma, t\right) \operatorname{coth} 2 t-\frac{\sigma^{\prime}(j) \sigma(j)}{\sinh 2 t} k^{m-n}\left(j, \sigma^{\prime}, \sigma, t\right)\right] \\
& \quad+(m-n-1) S^{m-n-1}\left(\sigma^{\prime}, \sigma, t\right),
\end{aligned}
$$

we have that

$$
\begin{aligned}
\sum_{i} k^{m-n-1}\left(i, \sigma^{\prime}, \sigma, t\right)= & \frac{2(m-n-1)}{\sinh ^{m-n-1} 2 t} \int_{0}^{t}\left[\sinh ^{m-n-1} 2 t^{\prime} \sum_{j} \frac{\sigma^{\prime}(j) \sigma(j)}{\sinh 2 t^{\prime}} k^{m-n}\left(j, \sigma^{\prime}, \sigma, t\right)\right. \\
& \left.-\left(\sinh ^{m-n-1} 2 t^{\prime}\right) S^{m-n-1}\left(\sigma^{\prime}, \sigma, t^{\prime}\right)\right] d t^{\prime}
\end{aligned}
$$


Since $R\left(k^{m-n}(j, \cdot, \cdot, t)\right) \leqq R(S)$ and $\sigma^{\prime}(j) \sigma(j) k^{m-n}\left(j, \sigma^{\prime}, \sigma, t\right)$ has the same super range as $k^{m-n}(j, \cdot, \cdot, t), R\left(k^{m-n-1}(i, \cdot, \cdot, t)\right) \leqq R(S)$. Hence $R\left(k^{n}(i, \cdot, \cdot, t) \leqq R(S)\right.$ for all $1 \leqq n \leqq m$. Therefore $R(k(i, \cdot, \cdot, t)) \leqq R(S)$.

Lemma 2.4. If $C(V)=C$ and $r(V)=R$, then

$$
C\left(h_{n}(i, t)\right) \leqq n(C-1)+1 \quad \text { and } \quad R\left(h_{n}(i, t)\right) \leqq n R .
$$

Proof. Prove by induction. Since $R(V)=r(V)$, the assertion holds for $n=1$ by Lemma 2.3. Suppose it is true for $m=1,2, \ldots, n-1$; then by Lemma 2.3 and Eq. (2.12b),

$$
\begin{aligned}
C\left(h_{n}(i, t)\right) & =C\left(\sum_{i}\left(\exp h_{0}(i, t)\right) P_{n}\left(h_{1}(i, t), \ldots, h_{n-1}(i, t)\right)\right) \\
& \leqq \sup _{i} \sup _{\substack{\sum_{l} n_{l}=n \\
n_{l} \geqq 1}}\left[C\left(\mathrm{~h}_{n_{l}}(\mathrm{i}, \mathrm{t})\right)-1\right]+1 \\
& \leqq \sup _{i} \sum_{l}\left[\left(\mathrm{n}_{l}(\mathrm{C}-1)+1\right)-1\right]+1 \\
& =n(C-1)+1 .
\end{aligned}
$$

Note that since the $h_{n_{l}}\left(i, \sigma^{\prime}, \sigma, t\right)$ 's contain a common factor $\sigma(i)$, $\left.C\left(P_{n}\right) \leqq \sup _{\sum n_{l}=n} \sum_{l}\left(C\left(h_{n_{l}}\right)-1\right)+1.\right]$ We also have that

$$
R\left(\sum_{i}\left(\exp h_{0}(i, t)\right) P_{n}\left(h_{1}(i, t), \ldots, h_{n-1}(i, t)\right)\right) \leqq n R .
$$

Thus by Lemma 2.3, we have

$$
R\left(h_{n}(i, t)\right) \leqq n R
$$

Lemma 2.5. Consider Eq. (2.22). Suppose $S$ is translation invariant and has cardinality $m \geqq 1$. Suppose that there exist constants $q_{n} \geqq 0(n=0,1,2, \ldots, m)$ and $p \geqq 0$ such that $\left\|S^{n}(t)\right\|_{0} \leqq|\Lambda| q_{n} t^{p}$. Then, under the hypotheses of Lemma 2.3,

$$
\left\|k^{n}(i, t)\right\|_{0} \leqq t^{p+1} q_{n}^{\prime}
$$

and

$$
\|k(i, t)\|_{0} \leqq t^{p+1} \sum_{n=1}^{m} q_{n}^{\prime} \leqq 2 t^{p+1}\left(\frac{m}{p+1}\right) \sum_{n=1}^{m} q_{n},
$$

for some constants $q_{n}^{\prime} \geqq 0, n=1,2, \ldots, m$. Moreover

$$
\left\|k(i, t)+k^{0}(i, t)\right\|_{0} \leqq 2 t^{p+1}\left(\frac{m+1}{p}\right) \sum_{n=0}^{m} q_{n}, \quad \text { if } \quad p>0 .
$$

Proof. Consider all the $m$-terms in Eq. (2.22) given by Eq. (2.26). By the translation invariance and the closure property $\left[\left\|k\left(i, \sigma^{\prime}, \sigma, t\right)\right\|_{0}\right.$ is translation invariant $]$, we get 


$$
\begin{aligned}
|\Lambda|\left\|k^{m}(i, t)\right\|_{0} & =\sum_{i}\left\|k^{m}(i, t)\right\|_{0}=\left\|\sum_{i} k^{m}(i, t)\right\|_{0} \\
& \leqq 2 m|\Lambda| \int_{0}^{t} \frac{t^{\prime m}}{t^{m}} q_{m} t^{\prime p} d t^{\prime}=\frac{2 m|\Lambda|}{m+p+1} q_{m} t^{p+1}
\end{aligned}
$$

where we used the fact that $\frac{\sinh 2 t^{\prime}}{\sinh 2 t} \leqq \frac{t^{\prime}}{t}$, for all $0<t^{\prime} \leqq t$. For $0<n<m$, Eq. (2.22) yields

$$
\sum_{i} k^{n}\left(i, \sigma^{\prime}, \sigma, t\right)=\frac{2 n}{\sinh ^{n} 2 t} \int_{0}^{t} \sinh ^{n} 2 t^{\prime}\left(\sum_{i} \frac{\sigma^{\prime}(i) \sigma(i) k^{n+1}\left(i, \sigma^{\prime}, \sigma, t\right)}{\sinh 2 t^{\prime}}-S^{n}\left(\sigma^{\prime}, \sigma, t^{\prime}\right)\right) d t^{\prime} .
$$

Thus, again by the translation invariance and the closure property,

$$
\left\|k^{n}(i, t)\right\|_{0} \leqq n \int_{0}^{t} \frac{t^{\prime n}}{t^{n}}\left(\frac{\left\|k^{n+1}\left(i, t^{\prime}\right)\right\|_{0}}{t^{\prime}}+2|\Lambda|^{-1}\left\|S^{n}\left(t^{\prime}\right)\right\|_{0}\right) d t^{\prime},
$$

where we used the fact that $(\sinh 2 t)^{-1} \leqq(2 t)^{-1}$, for $t>0$. This inequality gives generally, for $0<n \leqq m$,

$$
\left\|k^{n}(i, t)\right\|_{0} \leqq 2 t^{p+1} \sum_{r=n}^{m} q_{r} \prod_{s=0}^{r-n} \frac{n+s}{n+s+p+1} \equiv t^{p+1} q_{n}^{\prime} .
$$

Summing this inequality over $n$, we obtain

$$
\begin{aligned}
\|k(i, t)\|_{0} & =2 t^{p+1} \sum_{n=1}^{m} \sum_{r=n}^{m} q_{r} \prod_{s=0}^{r-n}\left(1+\frac{p+1}{n+s}\right)^{-1} \\
& \leqq 2 t^{p+1} \sum_{r=1}^{m} q_{r} \sum_{n=1}^{r} \prod_{s=0}^{r-n}\left(1+\frac{p+1}{m}\right)^{-1} \\
& \leqq 2 t^{p+1} \sum_{r=1}^{m} q_{r} \sum_{n=1}^{\infty}\left(1+\frac{p+1}{m}\right)^{-n} \\
& =2 t^{p+1}\left(\sum_{r=1}^{m} q_{r}\right) \frac{m}{p+1} .
\end{aligned}
$$

If $p>0$, special consideration of the $n=0$ terms gives

$$
\begin{aligned}
\left\|k^{0}(i, t)\right\|_{0} & \leqq \int_{0}^{t}\left(t^{\prime-1}\left\|k^{1}\left(i, t^{\prime}\right)\right\|_{0}+2|\Lambda|^{-1}\left\|S^{0}\right\|_{0}\right) d t^{\prime} \\
& \leqq \frac{2 t^{p+1}}{p+1}\left(\sum_{r=1}^{m} q_{r} \prod_{s=0}^{r-1} \frac{1+s}{1+s+p+1}+q_{0}\right) .
\end{aligned}
$$

Estimating $1+s$ by $2+s$ in the numerator of the products, inequalities (2.31a) and (2.31b) can be written as

$$
\left\|k^{n}(i, t)\right\|_{0} \leqq 2 t^{p+1} \sum_{r=n}^{m} q_{r} \prod_{s=0}^{r-n}\left(\frac{1+s+n}{1+s+n+p}\right), \quad n \geqq 0 .
$$

Summing this inequality over $n \geqq 0$, we obtain

$$
\left\|k(i, t)+k^{0}(i, t)\right\|_{0} \leqq 2 t^{p+1} \frac{m+1}{p} \sum_{n=0}^{m} q_{n} .
$$


We now apply these estimates to Eqs. (2.12a) and (2.12b). Since $V$ is independent of $t$ and has cardinality $C$, we get, by applying Lemma 2.5 to (2.12a), for all $1 \leqq m \leqq C$,

$$
\left\|h_{1}^{m}(i, t)\right\|_{0} \leqq q_{m}^{\prime} t, \quad \text { for some constant } \quad q_{m}^{\prime} \geqq 0,
$$

and

$$
\left\|h_{1}(i, t)\right\|_{0} \leqq \sum_{n=1}^{C} q_{n}^{\prime} t \leqq 2 C\|V\|_{0}|\Lambda|^{-1} t=B_{1} t,
$$

where $B_{1} \equiv 2 C\|V\|_{0}|\Lambda|^{-1}$.

For any fixed $\tau>0$, we can find a constant $A>1$ such that, by Eq. (2.11)

$$
\left\|\exp h_{0}(i, t)\right\|_{0}=\left|\frac{\cosh 2 t+1}{\sinh 2 t}\right| \leqq \frac{\dot{A}}{2 C t}
$$

for all $0<t \leqq \tau$. This will be used in the proof of the following lemma.

Lemma 2.6. Let $n \geqq 1$ and $0<t \leqq \tau$. Then

$$
\left\|h_{n}(i, t)\right\|_{0} \leqq B_{n} t^{n}
$$

where the $B_{n}$ 's are defined recursively by

$$
B_{n}=A P_{n}\left(B_{1}, B_{2}, \ldots, B_{n-1}\right), \quad n \geqq 2,
$$

and $B_{1}$ is defined by Eq. (2.33) while $A$ is given by Ineq. (2.34).

Proof. Prove by induction. By Eqs. (2.32) and Eq. (2.33), the result holds for $n=1$. Suppose that the lemma is true for $1,2, \ldots, n-1$, and that there exist constants $q_{m k} \geqq 0, m \geqq 1$, and $1 \leqq k \leqq n-1$ such that

$$
\left\|h_{k}^{m}(i, t)\right\|_{0} \leqq q_{m k} t^{k}, \quad m \geqq 1, \quad \text { and } \quad 1 \leqq k \leqq n-1,
$$

and

$$
\sum_{m \geqq 1} q_{m k} \leqq B_{k}, \quad 1 \leqq k \leqq n-1 .
$$

Then the source term in Eq. (2.12b) satisfies the estimate

$$
\begin{aligned}
|\Lambda|^{-1}\left\|\sum_{i}\left(\exp h_{0}(i, t)\right) P_{n}\left(h_{1}(i, t), \ldots, h_{n-1}(i, t)\right)\right\|_{0} & \leqq \frac{A}{2 C t} P_{n}\left(B_{1} t, B_{2} t^{2}, \ldots, B_{n-1} t^{n-1}\right) \\
& =\frac{A}{2 C} t^{n-1} P_{n}\left(B_{1}, B_{2}, \ldots, B_{n-1}\right),(2.38)
\end{aligned}
$$

by Ineq. (2.34), using the facts that $P_{n}\left(B_{1} t, \ldots, B_{n-1} t^{n-1}\right)$ is homogeneous in $t$ with degree $n$, and that $P_{n}$ is a polynomial with positive coefficients. Letting $F_{m}$ be the $m$-terms in

$$
\sum_{i} \exp \left(h_{0}(i, t)\right) P_{n}\left(h_{1}(i, t), \ldots, h_{n-1}(i, t)\right),
$$

we have that there exist constants $q_{n m} \geqq 0$ such that

$$
|\Lambda|^{-1}\left\|F_{m}\right\|_{0} \leqq q_{n m} t^{n-1}, \quad m \geqq 1,
$$


and that these $q_{n m}$ satisfy

$$
\sum_{m \geqq 1} q_{n m} \leqq(2 C)^{-1} A P_{n}\left(B_{1}, \ldots, B_{n-1}\right),
$$

since $\sum_{m \geqq 1}\left\|F_{m}\right\|_{0}=\left\|\sum_{m \geqq 1} F_{m}\right\|_{0}$ by the definition of the norm. By Lemmas 2.3 and 2.4 , and the definition of $B_{n}$, we have

$$
\begin{aligned}
\left\|h_{n}(i, t)\right\|_{0} & \leqq 2 t^{n} \frac{C\left(h_{n}\right)}{n} \frac{B_{n}}{2 C} \\
& \leqq 2 t^{n} \frac{n(C-1)+1}{n} \frac{B_{n}}{2 C} \\
& \leqq B_{n} t^{n} . \quad \square
\end{aligned}
$$

Lemma 2.7. The $B_{n}$ 's are the coefficients for an analytic function $B(\varepsilon)=\sum_{n=1}^{\infty} \varepsilon^{n} B_{n}$, which is convergent in a neighborhood of the origin.

Proof. Define $B(\varepsilon)$ implicity by $(B(0)=0)$

$$
F(B, \varepsilon) \equiv A \exp B(\varepsilon)-(A+1) B(\varepsilon)-A+B_{1} \varepsilon=0 .
$$

The function $B(\varepsilon)$ exists and is analytic in a neighborhood of the origin since $\frac{\partial F}{\partial B}(0,0) \neq 0$ and $B(0)=0$. Rearranging Eq. (2.39), we obtain

$$
B(\varepsilon)=A(\exp B(\varepsilon)-B(\varepsilon))-A+B_{1} \varepsilon
$$

Expanding $B(\varepsilon)=\sum_{n=1}^{\infty} \varepsilon^{n} B_{n}^{\prime}$, we obtain recursively, [see Eq. (2.13)], $B_{1}^{\prime}=B_{1}$, and

$$
B_{n}^{\prime}=A P_{n}\left(B_{1}, \ldots, B_{n-1}\right)=B_{n}, \quad n \geqq 2 .
$$

We now complete the proof of the theorem.

i) Since

$$
\begin{aligned}
h_{0}\left(i, \sigma^{\prime}, \sigma, t\right)= & \log \frac{\cosh 2 t-\sigma^{\prime}(i) \sigma(i)}{\sinh 2 t} \\
= & \log \operatorname{coth} 2 t-\sum_{n=1}^{\infty} \frac{1}{n}\left(\frac{\sigma^{\prime}(i) \sigma(i)}{\cosh 2 t}\right)^{n}, \\
& \left\|h_{0}(i, t)\right\|_{0} \leqq|\log \operatorname{coth} 2 t|+\sum_{n=1}^{\infty} \frac{1}{n} \frac{1}{\cosh ^{n} 2 t} \rightarrow 0, \quad t \rightarrow \infty .
\end{aligned}
$$

Hence for each $\delta>0$, there exists a $\tau>0$ such that for $t \geqq \tau$

$$
\left\|h_{0}(i, t)\right\|_{0} \leqq \delta / 2 \text {. }
$$

By Lemmas 2.6 and 2.7, there exists an $\varepsilon_{1}>0$ such that for $\varepsilon \leqq \varepsilon_{1}$

$$
\sum_{n=1}^{\infty} \varepsilon^{n}\left\|h_{n}(i, t)\right\|_{0} \leqq \sum_{n=1}^{\infty} \varepsilon^{n} \tau^{n} B_{n}<\delta / 2
$$




\section{Therefore}

$$
\sum_{n=0}^{\infty} \varepsilon^{n}\left\|h_{n}(i, t)\right\|_{0}<\delta / 2+\delta / 2=\delta
$$

ii) It is easy to see that if $n<\frac{d(i, \partial \Lambda)}{R}$ and $\Lambda \subset \Lambda^{\prime}$, then $h_{\Lambda k}\left(i, \sigma^{\prime}, \sigma, t\right)$ and $h_{\Lambda^{\prime} k}\left(i, \sigma^{\prime}, \sigma, t\right), k=1,2, \ldots, n$, satisfy the same differential equations and hence are equal. Thus

$$
\begin{aligned}
\left\|h_{\Lambda}(i, t, \varepsilon)-h_{\Lambda^{\prime}}(i, t, \varepsilon)\right\|_{0} & \leqq \sum_{n \geqq \frac{d(i, \partial \Lambda)}{R}} \varepsilon^{n}\left\|h_{\Lambda k}(i, t)-h_{\Lambda^{\prime} k}(i, t)\right\|_{0} \\
& \leqq \sum_{n \geqq \frac{d(i, \partial \Lambda)}{R}} 2 \varepsilon^{n} B_{n} t^{n}=O\left(\varepsilon^{d(i, \partial \Lambda) / R}\right) .
\end{aligned}
$$

iii) From the statement i) of the theorem, it is easy to see that there is an $\varepsilon_{1}>0$ such that for $0<\varepsilon \leqq \varepsilon_{0}<\varepsilon_{1}$,

and [see Eq. (2.16)]

$$
\sum_{n \leqq \frac{|i-j|}{R}} \varepsilon^{n}\left\|h_{\Lambda n}(i, t)\right\|_{0}<\delta\left(\frac{\varepsilon}{\varepsilon_{1}}\right)^{\frac{|i-j|}{R}}
$$

$$
\sum_{j \in \mathbb{Z}^{\nu}} \delta\left(e \cdot\left(\frac{\varepsilon}{\varepsilon_{1}}\right)^{1 / R}\right)^{|i-j|}<\infty
$$

\section{References}

1. Ginibre, J.: Existence of phase transitions for quantum lattice systems. Commun. Math. Phys. 14, 205-234 (1969)

2. Kirkwood, J.R.: Ph. D. dissertation, University of Virginia (1982)

3. Ruelle, D.: Statistical mechanics. New York: Benjamin 1969

4. Holley, R.A., Stroock, D. : Applications of the stochastic Ising model to the Gibbs states. Commun. Math. Phys. 48, 249-265 (1976)

5. Gross, L.: Decay of correlations in classical lattice models at high temperature. Commun. Math. Phys. 68, 9-27 (1979)

6. Malyshev, V.A.: Uniform cluster estimates for lattice models. Commun. Math. Phys. 64, 131-157 (1979)

7. Cammarota, C.: Decay of correlations for infinite range interactions in unbounded spin systems. Commun. Math. Phys. 85, 517-528 (1982)

8. Hagedorn, R., Rafelski, J.: Analytic structure and explicit solution of an important implicit equation. Commun. Math. Phys. 83, 563-578 (1982)

9. Kirkwood, J.R., Thomas, L.E.: Expansions and phase transitions for the ground state of quantum Ising lattice systems. Commun. Math. Phys. 88, 569-580 (1983)

10. Fröhlich, J., Lieb, E.: Phase transitions in an isotropic spin system. Commun. Math. Phys. 60, 233-267 (1978)

11. Elliott, R.J., Pfeuty, P., Wood, C.: Ising model with a transverse field. Phys. Rev. Lett. 25, 443-446 (1970)

12. Pfeuty, P.: The one-dimensional Ising model with a transverse field. Ann. Phys. (NY) 57, 79-90 (1970)

Communicated by J. Fröhlich

Received April 14, 1983; in revised form June 19, 1983 
